\title{
Identifying Nonacademic Behaviors Associated With Post-School Employment and Education
}

Career Development and Transition for Exceptional Individuals 36(3) 174-187

(C) Hammill Institute on Disabilities 2012 Reprints and permissions: sagepub.com/journalsPermissions.nav DOI: 10.1 | $77 / 2$ | 65 | $434|2468| 47$ cdtei.sagepub.com

(SSAGE

\author{
Amber E. McConnell, PhD', James E. Martin, PhD', Chen Ya Juan, PhD', \\ Maeghan N. Hennessey, PhD', Robert A. Terry, PhD', Nidal A. el-Kazimi, PhD³, \\ Tammy C. Pannells, $\mathbf{P h D}^{4}$, and Donna M. Willis, BA'
}

\begin{abstract}
We conducted an analysis of the secondary transition qualitative and quantitative research literature to build comprehensive constructs and lists of student nonacademic behaviors associated with post-high school employment and education. From a pool of 83 initial quantitative and qualitative studies, 35 met the inclusion criteria, and the analysis of these yielded 10 constructs of student nonacademic behaviors associated with post-school education and employment for high school students with mild to moderate disabilities. The constructs are presented along with lists of representative behaviors, implications for their use are discussed, and the next steps in building a new transition assessment using these constructs and behaviors are described.
\end{abstract}

\section{Keywords}

transition assessment, employment, postsecondary education, non-academic skills, transition education

The purpose of Individuals With Disabilities Education Act (IDEA; 2004) clearly indicates special educators need to prepare students for post-high school education and employment, and IDEA 2004 requires Individualized Education Programs (IEPs) for students of transition age contain postsecondary employment, postsecondary education, and as needed, independent living goals (Kochhar-Bryant, Shaw, \& Izzo, 2007). Annual transition goals operationalize skills students need to learn to enable them to attain their postsecondary goals (Test, Aspel, \& Everson, 2006). Benz, Lindstrom, and Yovanoff (2000) found that basic academic skills, such as reading, writing, and arithmetic, alone are insufficient to enable students with disabilities to attain their post-school goals.

The Institute for Education Sciences considers six types of transition outcomes: (a) behavioral, (b) social, (c) communicative, (d) functional, (e) occupational, and (f) basic academic skills (Institute of Education Sciences, 2012). Basic academic skills, such as reading, spelling, and math calculation skills, are often evaluated by numerous academic assessments, and annual IEP goals may be written based on the results to enable progress toward academic outcomes. Using this same logic, IEP team members should consider building annual transition goals centered on nonacademic transition outcomes areas, which include behavioral, social, communicative, functional, and operational skills.
Test, Fowler, et al. (2009) gleaned 32 evidence-based secondary level transition education practices from the research literature that improved specific student skills. For example, methods for teaching life and purchasing skills had strong levels of evidence of their effectiveness. Strategies for teaching students to become actively involved in their IEP meetings had moderate levels of effectiveness. They identified numerous effective teaching strategies, but because the experimental studies used to create the practices did not include data on the long-term impact of skills acquired, they do not indicate whether the learned skills were associated with improved post-school employment or education outcomes.

In response to this limitation, Test, Mazzotti, et al. (2009) evaluated correlational research to identify in-school predictors of positive post-school education, employment, and/or

\footnotetext{
'University of Oklahoma, Norman, USA

${ }^{2} \mathrm{Hsin}$ Sheng College of Medical Care and Management, Taoyuan County, Taiwan

${ }^{3}$ Shafallah Center for Children With Disabilities, Doha, Qatar

${ }^{4}$ Health Statistics Branch-County Survey Section, Little Rock, AR, USA

Corresponding Author:

Amber E. McConnell, University of Oklahoma, Zarrow Center for Learning Enrichment, 338 Cate Center Drive, Room 190, Norman, OK 73019, USA.

Email:ambermcc@ou.edu
} 
independent living outcomes for students with disabilities. Their work yielded 16 predictors of positive student postschool employment, education, and independent living outcomes. Twelve predictors involved programs, services, placements, or processes, and four categories were student behaviors or experiences, including paid employment, selfadvocacy and self-determination, self-care, and social skills. Except for paid employment, the remaining three broadly defined categories did not identify specific teachable student behaviors associated with positive post-school outcomes.

The previously described reviews advanced the field by identifying effective instructional approaches and predictor variables, but did not develop comprehensive definitional constructs and listings of specific student behaviors and experiences associated with improved post-school education and employment. Because of this, there is a need to identify nonacademic student behaviors and experiences to provide IEP teams student behaviors associated with postschool education and employment that can easily be used to develop annual transition goals.

Juan (2008) was one of the first researchers to identify research-based nonacademic student behaviors and experiences associated with improved post-school education and employment outcomes for students with disabilities. Her review of the literature identified 41 behaviors and experiences of high school students with disabilities that contributed or should contribute to involvement in post-school employment or education. Juan grouped these 41 behaviors into 12 domains: (a) desires, (b) strengths, (c) disability awareness, (d) use of support systems, (e) social skills, (f) making positive choices, (g) goals, (h) limits, (i) persistence, $(\mathrm{j})$ coping skills, $(\mathrm{k})$ proactive involvement, and (l) transition education involvement. Each domain included several specific student behaviors, but Juan did not develop construct definitions.

Using Juan's (2008) initial framework, we sought to develop construct definitions of nonacademic student behaviors and experiences associated with post-school education and employment and form a list of specific teachable skills derived from the research. Thus, the purpose of this study is to build upon Juan's initial findings and the work of Test, Mazzotti, et al. (2009), and review the research literature to identify additional nonacademic student behaviors associated with post-school employment and further education of former students with mild to moderate disabilities. We will cluster the identified studies together, develop cluster definitions, and create exemplar lists of teachable behaviors.

\section{Method}

To undertake this study, several experienced educational researchers examined the research literature in an iterative process to develop constructs defining student behaviors associated with employment and further education after high school. The following describes the process used to develop constructs and exemplar behaviors.

\section{The Research Team}

A seven-member research team was strategically assembled to develop a new transition assessment based on current secondary transition research. The team collaboratively used a consensus decision-making process to identify research studies, review the studies, and build constructs and associated lists of behaviors from which the assessment will be written. The research team consisted of (a) a professor of special education knowledgeable about transition education, (b) an assistant professor of educational psychology specializing in statistical analysis and assessment development who was also a former high school educator, (c) an associate professor of psychology specializing in statistical analysis and assessment development, (d) a postdoctoral educational psychology researcher who was a former educator, (e) an advanced special education doctoral student knowledgeable about transition and who recently worked as a secondary special education teacher, $(\mathrm{g})$ an advanced special education doctoral student knowledgeable about transition and who previously worked as a vocational educator of students with special needs, and (g) a psychology undergraduate student who worked full-time at the research center where this project was completed and who provided input from a parent's perspective.

\section{Identification of Research Studies}

We employed a five-step process to find studies that identified the nonacademic behaviors of students with disabilities associated with successful participation in post-school employment and education as identified by authors through correlational, qualitative, and descriptive data analyses. For the behavior or skill to be considered "associated" with post-school employment or education, we looked for relations to education and employment in correlational research, skills, or behaviors reported to be characteristic of individuals with disabilities engaging in post-school education and employment in qualitative research (Spradley, 1979), and identified variables that described post-school employment and education in descriptive research. First, we obtained copies of the studies identified by Juan (2008). Second, we acquired the correlational studies used by Test, Mazzotti, et al. (2009) who identified behavioral predictors of improved post-school outcomes. Third, we sought additional studies by conducting an online search using "EBSCOhost" with keywords and phrases, including "post-school success," "follow-up studies," "transition," and "students with disabilities." Fourth, we used the references of the obtained manuscripts to find additional studies. Fifth, we examined the table of contents of special 
education and transition journals from the past 3 years (2009-2012) to identify studies not yet included or missed in online database searches. These five steps yielded 83 initial studies published between 1985 and the present that identified behaviors associated with successful participation in post-school employment and education for students with disabilities. We did not impose date criteria when searching for the studies because we wanted an inclusive set of studies. The seven-member research team then read and applied the inclusion and exclusion criteria described below to determine studies to be used.

Because our focus with this study was on developing specific construct definitions, we utilized the complete transition education literature to comprehensively identify nonacademic behaviors associated with post-high school participation in education and employment of former students with mild to moderate disabilities. Our inclusion and exclusion criteria enabled us to obtain a variety of studies to create a representative and inclusive synthesis of what the entire spectrum of transition education research has identified. Thus, we examined mixed-method studies, pre-post studies, qualitative studies, and correlational studies to identify as many student behaviors as possible. Using a combination of qualitative and quantitative data allowed for rich and complete definitions of constructs and identified behaviors from multiple perspectives, including those of individuals with disabilities. Passing the peer-review process and being published served as our initial study quality indicator.

\section{Inclusion and Exclusion Criteria}

The purpose of the literature analysis was to build constructs based on student nonacademic behaviors associated with participation in post-school employment and education. From the constructs, exemplar lists of student behaviors would be developed. Thus, this is not a comprehensive literature review that found every study that identified the same behavior time after time. Three criteria were applied to determine whether a quantitative or qualitative research study was included or excluded. First, the manuscript had to clearly identify student behaviors associated with post-high school employment or education. The behaviors identified in a study needed to be specific enough to be easily converted into annual transition goals. For example, the study by Sands, Spencer, Gliner, and Swaim (1999), which only identified in-school student success indicators, was excluded because the in-school behaviors were not associated with post-high school employment or further education. Behaviors identified by Goldberg, Higgins, Raskind, and Herman (2003) were included because the qualitative study identified specific attributes that provided a richer definition of constructs identified by quantitative studies, such as "the ability to compartmentalize their disability" (p. 226).
Second, a study had to include individuals identified by its authors as having mild to moderate disabilities. We excluded studies that focused on students without disabilities, as well as studies that only included individuals with severe or profound intellectual disabilities (mental retardation) because our target population consisted of students with mild to moderate disabilities, and the lack of research identifying specific behaviors associated with maintaining employment and enrollment in further education for students with severe or profound intellectual disabilities. Third, studies that only identified academic indicators of improved post-school outcomes, such as grades, passing state mandated tests, and inclusion in general education setting, were excluded for two primary reasons. First, Benz et al. (2000) found that academic skills alone are insufficient for improved post-school outcomes. Many standardized assessments already focus on academic skills. Second, the extensive focus on academic skills and academic assessment already present in schools and within the IEP does not seem to be sufficiently improving the post-school outcomes of students with disabilities (Rusch, Hughes, Agran, Martin, \& Johnson, 2009). Thus, studies such as the one completed by Heal and Rusch (1994) that focused on academic skills, grade point average (GPA), and the receipt of a diploma as predictors of improved post-school outcomes were excluded.

\section{Building Constructs and Associated Lists of Behaviors and Experiences}

The research team used a consensus decision-making process to build the constructs where ideas were discussed and debated until everyone agreed. The research team implemented a seven-step process over 4 months to define constructs and build the list of post-school student behaviors associated with participation in employment and postsecondary education. First, the research team read the studies and individually applied the inclusion and exclusion criteria. Second, the research team discussed each study and jointly applied the inclusion and exclusion criteria to decide whether a study was to be examined in more depth, and we grouped them into common clusters based on the behavior(s) each identified. Third, each group member reread approximately half of the included studies, then met and determined by consensus initial construct titles and clusters of articles associated with each construct. This process ensured that across the team, at least three team members had read and examined each study, and the other team members were familiar with the study when a specific study was discussed.

Fourth, all research team members jointly composed the "Disability Awareness" construct definition from the list of initial cluster of studies as an example of how to build additional constructs. The research team also composed a list of associated behaviors from the literature to operationalize the 
construct. Prior to the initial construct-building meeting, each research team member reread the cluster of disability awareness studies. During a consensus discussion, the team agreed that 11 studies had identified disability awareness as an indicator of post-school employment and enrollment in postsecondary education. Using these studies, the team gleaned language to build the disability awareness construct, and then constructed a list of specific student behaviors associated with disability awareness directly from the studies.

Fifth, the team divided into two subgroups to create definitions for each remaining construct. To do this, the entire research team identified weekly a set of studies for the two subgroups to read. Each subgroup read and studied the identified set of articles and then met independently of the other group to develop a draft behavior construct and a list of associated student behaviors according to the process described in Step 4.

Sixth, the two subgroups reconvened weekly and each presented the tentative construct definitions and lists of behaviors developed that week to the entire team. The constructs and behavior lists were adjusted based on comments and suggestions based on the literature from the entire research team until consensus constructs and behavior lists were developed. Once a few studies identified the same behavior, our focus shifted to identifying additional behaviors. Thus, we are confident the analysis produced a comprehensive list of student behaviors associated with post-school participation in employment and education, but not every study associated with a specific behavior was listed or used. Seventh, the first author went back through each of the constructs, matched the citations to the studies to make certain they conformed to the inclusion criteria, and made additions or deletions as needed. The second author then independently verified the changes. The two disagreements were discussed and resolved.

\section{Results}

Only 35 studies out of the 83 initial studies met the inclusion criteria. Our comprehensive literature review of these 35 studies resulted in 10 constructs and exemplar behaviors associated with positive post-school outcomes of former students with mild to moderate disabilities. These constructs are (a) knowledge of strengths and limitations, (b) actions related to strengths and limitations, (c) disability awareness, (d) employment, (e) goal setting and attainment, (f) persistence, (g) proactive involvement, (h) self-advocacy, (i) supports, and (j) utilization of resources. See Table 1 for a complete list of studies and type of analyses used to identify each behavior, organized by constructs.

\section{Knowledge of Strengths and Limitations}

Numerous studies found individuals with disabilities who know their personal strengths and limitations are more likely to successfully participate in employment and education after high school (Aune, 1991; Goldberg et al., 2003; Higgins, Raskind, Goldberg, \& Herman, 2002; Lindstrom, Doren, \& Miesch, 2011; Madaus, 2006; McNulty, 2003; Raskind, Goldberg, Higgins, \& Herman, 1999, 2002; Sarver, 2000). Students may not use correct terminology, yet need to be aware of their strengths and limitations in adult world settings and must be able to communicate these strengths and limitations to others (Gerber, Ginsberg, \& Reiff, 1992; Higgins et al., 2002; Madaus, 2006; Raskind et al., 2002; Sarver, 2000, Skinner, 2004), and make job choices based on their strengths (Lindstrom et al., 2011). Individuals who identify situations and tasks where they will most likely be successful, as well as unsuccessful, are more likely have positive post-school employment and education outcomes (Gerber et al., 1992).

\section{Actions Related to Strengths and Limitations}

Once students are aware of their strengths and limitations, they must act upon their knowledge by seeking situations where they maximize strengths and minimize limitations (Aune, 1991; Goldberg et al., 2003; Greenbaum, Graham, \& Scales, 1995; Madaus, 2006; McNulty, 2003; Sarver, 2000;). Students must actively seek situations to use their strengths in school and in the community (Gerber et al., 1992; Goldberg et al., 2003; McNulty, 2003). For example, if a student has a disability in the area of written expression, he or she may choose to become a club photographer rather than the club secretary. Students need to develop skills and strategies to compensate for limitations, such as lip reading, memory aids, and use of assistive technology (Gerber et al., 1992; Higgins et al., 2002; Raskind et al., 2002; Skinner, 2004). Students may create new strategies to accomplish tasks that are difficult (Higgins et al., 2002: Raskind et al., 2002; Skinner, 2004). Individuals who successfully engage in post-school education and employment consider their limitations when choosing goals that can be accomplished (Thoma \& Getzel, 2005) and do not choose careers that require frequent use of their limitations (Gerber et al., 1992).

\section{Disability Awareness}

Self-understanding of a student's disability provides the foundation for all transition skills (Aune, 1991). Individuals with disabilities who participate in employment and further education are aware of their disability, yet the disability does not define them (Goldberg et al., 2003; Raskind et al., 1999; Raskind et al., 2002; Sarver, 2000), and know how their disability affects how they learn (Skinner, 2004). Some individuals consider their disability as a positive aspect of life (Higgins et al., 2002). Disability awareness begins with the student understanding the definition of the word "disability" (Aune, 1991; Greenbaum et al., 1995), followed by an understanding of the challenges they will face due to the 
Table I. Constructs, Behaviors, and Associated Studies.

\begin{tabular}{|c|c|c|}
\hline Specific behavior & Associated studies & Study design \\
\hline \multicolumn{3}{|l|}{ Construct I: Knowledge of strengths and limitations } \\
\hline $\begin{array}{l}\text { Student tells someone about his or her } \\
\text { strengths. }\end{array}$ & $\begin{array}{l}\text { Higgins, Raskind, Goldberg, \& Herman, } 2002 \\
\text { Lindstrom, Doren, \& Miesch, 20I I } \\
\text { Madaus, } 2006 \\
\text { Raskind, Goldberg, Higgins, \& Herman, } 1999\end{array}$ & $\begin{array}{l}\text { Qualitative Ethnography } \\
\text { Qualitative Case Study } \\
\text { Qualitative Inductive } \\
\text { Correlation Descriptive }\end{array}$ \\
\hline $\begin{array}{l}\text { Student tells someone what he or she does } \\
\text { well. }\end{array}$ & $\begin{array}{l}\text { Goldberg, Higgins, Raskind, \& Herman, } 2003 \\
\text { McNulty, } 2003 \\
\text { Raskind, Goldberg, Higgins, \& Herman, } 2002\end{array}$ & $\begin{array}{l}\text { Qualitative Ethnography } \\
\text { Qualitative Narrative } \\
\text { Qualitative }\end{array}$ \\
\hline Student tells someone his or her limitations. & $\begin{array}{l}\text { Aune, I991 } \\
\text { Gerber, Ginsberg, \& Reiff, } 1992\end{array}$ & $\begin{array}{l}\text { Descriptive } \\
\text { Qualitative Ethnography }\end{array}$ \\
\hline $\begin{array}{l}\text { Student tells someone what he or she has } \\
\text { trouble doing. }\end{array}$ & Gerber et al., 1992 & Qualitative Ethnography \\
\hline $\begin{array}{l}\text { Student expresses accurate information } \\
\text { about his or her academic strengths. }\end{array}$ & $\begin{array}{l}\text { Aune, I99I } \\
\text { Higgins et al., } 2002 \\
\text { Raskind et al., } 2002\end{array}$ & $\begin{array}{l}\text { Descriptive } \\
\text { Qualitative Ethnography } \\
\text { Qualitative }\end{array}$ \\
\hline $\begin{array}{l}\text { Student expresses accurate information } \\
\text { about his or her academic limitations. }\end{array}$ & $\begin{array}{l}\text { Aune, I99I } \\
\text { Gerber et al., I } 992 \\
\text { Higgins et al., } 2002 \\
\text { Madaus, } 2006 \\
\text { Sarver, } 2000 \\
\text { Skinner, } 2004\end{array}$ & $\begin{array}{l}\text { Descriptive } \\
\text { Qualitative Ethnography } \\
\text { Qualitative Ethnography } \\
\text { Qualitative Inductive } \\
\text { Correlation } \\
\text { Qualitative Constant Comparison }\end{array}$ \\
\hline $\begin{array}{l}\text { Student identifies academic situations when } \\
\text { assistance is needed. }\end{array}$ & $\begin{array}{l}\text { Aune, I991 } \\
\text { Gerber et al., } 1992 \\
\text { Higgins et al., } 2002\end{array}$ & $\begin{array}{l}\text { Descriptive } \\
\text { Qualitative Ethnography } \\
\text { Qualitative Ethnography }\end{array}$ \\
\hline $\begin{array}{l}\text { Student identifies academic situations where } \\
\text { he or she will likely experience success. }\end{array}$ & $\begin{array}{l}\text { Aune, I991 } \\
\text { Gerber et al., } 1992 \\
\text { Goldberg et al., } 2003 \\
\text { McNulty, } 2003\end{array}$ & $\begin{array}{l}\text { Descriptive } \\
\text { Qualitative Ethnography } \\
\text { Qualitative Ethnography } \\
\text { Qualitative Narrative }\end{array}$ \\
\hline \multicolumn{3}{|c|}{ Construct 2: Actions related to strengths and limitations } \\
\hline $\begin{array}{l}\text { Student finds situations to use his or her } \\
\text { strengths. }\end{array}$ & $\begin{array}{l}\text { Gerber et al., I } 992 \\
\text { Greenbaum, Graham, \& Scales, } 1995 \\
\text { Goldberg et al., } 2003 \\
\text { McNulty, } 2003\end{array}$ & $\begin{array}{l}\text { Qualitative Ethnography } \\
\text { Qualitative Descriptive } \\
\text { Qualitative Ethnography } \\
\text { Qualitative Narrative }\end{array}$ \\
\hline $\begin{array}{l}\text { Student is aware of his or her strengths, } \\
\text { and seeks situations where strengths are } \\
\text { utilized. }\end{array}$ & $\begin{array}{l}\text { Higgins et al., } 2002 \\
\text { Madaus, } 2006 \\
\text { Sarver, } 2000 \\
\text { Raskind et al., } 2002\end{array}$ & $\begin{array}{l}\text { Qualitative Ethnography } \\
\text { Qualitative Inductive } \\
\text { Correlation } \\
\text { Qualitative }\end{array}$ \\
\hline $\begin{array}{l}\text { Student finds situations where his or her } \\
\text { limitations are minimized. }\end{array}$ & $\begin{array}{l}\text { Aune, I99I } \\
\text { Gerber et al., I992 } \\
\text { Goldberg et al., } 2003 \\
\text { McNulty, } 2003 \\
\text { Thoma \& Getzel, } 2005\end{array}$ & $\begin{array}{l}\text { Descriptive } \\
\text { Qualitative Ethnography } \\
\text { Qualitative Ethnography } \\
\text { Qualitative Narrative } \\
\text { Qualitative }\end{array}$ \\
\hline $\begin{array}{l}\text { Student knows his or her weaknesses and } \\
\text { considers the weaknesses when making } \\
\text { choices. }\end{array}$ & $\begin{array}{l}\text { Gerber et al., } 1992 \\
\text { Madaus, } 2006 \\
\text { Raskind et al., } 2002 \\
\text { Sarver, } 2000 \\
\text { Skinner, } 2004\end{array}$ & $\begin{array}{l}\text { Qualitative Ethnography } \\
\text { Qualitative Inductive } \\
\text { Qualitative } \\
\text { Correlation } \\
\text { Qualitative Constant Comparison }\end{array}$ \\
\hline $\begin{array}{l}\text { Student creates new strategies to } \\
\text { compensate for his or her limitations. }\end{array}$ & $\begin{array}{l}\text { Aune, I991 } \\
\text { Higgins et al., } 2002 \\
\text { Raskind et al., } 2002 \\
\text { Skinner, } 2004\end{array}$ & $\begin{array}{l}\text { Descriptive } \\
\text { Qualitative Ethnography } \\
\text { Hierarchical Regression } \\
\text { Qualitative Constant Comparison }\end{array}$ \\
\hline \multicolumn{3}{|l|}{ Construct 3: Disability awareness } \\
\hline $\begin{array}{l}\text { Student expresses an understanding of the } \\
\text { word "disability." }\end{array}$ & $\begin{array}{l}\text { Aune, I991 } \\
\text { Greenbaum et al., } 1995\end{array}$ & $\begin{array}{l}\text { Descriptive } \\
\text { Qualitative Descriptive }\end{array}$ \\
\hline
\end{tabular}


Table I. (continued)

\begin{tabular}{|c|c|c|}
\hline Specific behavior & Associated studies & Study design \\
\hline $\begin{array}{l}\text { Student tells someone he or she has a } \\
\text { disability. }\end{array}$ & $\begin{array}{l}\text { Aune, I99I } \\
\text { Gerber et al., I } 992 \\
\text { Gerber, Price, Mulligan, \& Shessel, } 2004 \\
\text { Higgins et al., } 2002 \\
\text { Raskind et al., } 1999\end{array}$ & $\begin{array}{l}\text { Descriptive } \\
\text { Qualitative Ethnography } \\
\text { Qualitative Case Study } \\
\text { Qualitative Ethnography } \\
\text { Correlation and Descriptive }\end{array}$ \\
\hline $\begin{array}{l}\text { Student tells someone accurate information } \\
\text { about his or her disability. }\end{array}$ & $\begin{array}{l}\text { Aune, I99I } \\
\text { Gerber et al., } 1992 \\
\text { Greenbaum et al., } 1995 \\
\text { Higgins et al., } 2002 \\
\text { Thoma \& Getzel, } 2005\end{array}$ & $\begin{array}{l}\text { Descriptive } \\
\text { Qualitative Ethnography } \\
\text { Qualitative Descriptive } \\
\text { Qualitative Ethnography } \\
\text { Qualitative }\end{array}$ \\
\hline $\begin{array}{l}\text { Student uses the least stigmatizing disability } \\
\text { label that results in getting most support. }\end{array}$ & $\begin{array}{l}\text { Gerber et al., } 2004 \\
\text { Higgins et al., } 2002 \\
\text { Sarver, } 2000\end{array}$ & $\begin{array}{l}\text { Qualitative Ethnography } \\
\text { Qualitative Ethnography } \\
\text { Correlation }\end{array}$ \\
\hline $\begin{array}{l}\text { Student expresses types of accommodations } \\
\text { or supports needed for his or her disability. }\end{array}$ & $\begin{array}{l}\text { Aune, 1991 } \\
\text { Gerber et al., } 1992 \\
\text { Greenbaum et al., } 1995 \\
\text { Higgins et al., } 2002 \\
\text { Skinner, } 2004\end{array}$ & $\begin{array}{l}\text { Descriptive } \\
\text { Qualitative Ethnography } \\
\text { Qualitative Descriptive } \\
\text { Qualitative Ethnography } \\
\text { Qualitative Constant } \\
\text { Comparison }\end{array}$ \\
\hline $\begin{array}{l}\text { Student views disability as only one aspect } \\
\text { of his or her life. }\end{array}$ & $\begin{array}{l}\text { Goldberg et al., } 2003 \\
\text { Higgins et al., } 2002 \\
\text { Raskind et al., } 1999 \\
\text { Raskind et al., } 2002\end{array}$ & $\begin{array}{l}\text { Qualitative Ethnography } \\
\text { Qualitative Ethnography } \\
\text { Correlation and Descriptive } \\
\text { Qualitative }\end{array}$ \\
\hline $\begin{array}{l}\text { Student views disability as a positive aspect } \\
\text { of his or her life. }\end{array}$ & Higgins et al., 2002 & Qualitative Ethnography \\
\hline $\begin{array}{l}\text { Student explains to friends that he or she } \\
\text { receives special education services. }\end{array}$ & Higgins et al., 2002 & Qualitative Ethnography \\
\hline $\begin{array}{l}\text { Student talks to parents about his or her } \\
\text { disability. }\end{array}$ & $\begin{array}{l}\text { Aune, 1991 } \\
\text { Higgins et al., } 2002\end{array}$ & $\begin{array}{l}\text { Descriptive } \\
\text { Qualitative Ethnography }\end{array}$ \\
\hline \multicolumn{3}{|l|}{ Construct 4: Persistence } \\
\hline $\begin{array}{l}\text { Student views not giving up in school as } \\
\text { important. }\end{array}$ & $\begin{array}{l}\text { Goldberg et al., } 2003 \\
\text { Raskind, I } 999 \\
\text { Sarver, } 2000\end{array}$ & $\begin{array}{l}\text { Qualitative Ethnography } \\
\text { Qualitative } \\
\text { Correlation }\end{array}$ \\
\hline $\begin{array}{l}\text { Student keeps working until he or she } \\
\text { accomplishes a goal. }\end{array}$ & $\begin{array}{l}\text { Gerber et al., } 1992 \\
\text { Goldberg et al., } 2003 \\
\text { Greenbaum et al., } 1995 \\
\text { Skinner, } 2004\end{array}$ & $\begin{array}{l}\text { Qualitative Case Study } \\
\text { Qualitative Ethnography } \\
\text { Qualitative Descriptive } \\
\text { Qualitative Constant Comparison }\end{array}$ \\
\hline $\begin{array}{l}\text { Student utilizes different strategies as } \\
\text { needed to continue staying on task. }\end{array}$ & Goldberg et al., 2003 & Qualitative Ethnography \\
\hline $\begin{array}{l}\text { Student keeps working to achieve a goal, } \\
\text { even when it becomes hard. }\end{array}$ & $\begin{array}{l}\text { Gerber et al., } 1992 \\
\text { Goldberg et al., } 2003 \\
\text { Raskind et al., } 2002\end{array}$ & $\begin{array}{l}\text { Qualitative Case Study } \\
\text { Qualitative Ethnography } \\
\text { Qualitative }\end{array}$ \\
\hline $\begin{array}{l}\text { Student learns from mistakes and does } \\
\text { better next time. }\end{array}$ & $\begin{array}{l}\text { Greenbaum et al., } 1995 \\
\text { Goldberg et al., } 2003\end{array}$ & $\begin{array}{l}\text { Qualitative Descriptive } \\
\text { Qualitative Ethnography }\end{array}$ \\
\hline \multicolumn{3}{|l|}{ Construct 5: Proactive involvement } \\
\hline $\begin{array}{l}\text { Student maintains one or more good } \\
\text { friendships. }\end{array}$ & $\begin{array}{l}\text { Doren \& Benz, } 1998 \\
\text { Goldberg et al., } 2003\end{array}$ & $\begin{array}{l}\text { Logistic Regression } \\
\text { Qualitative Ethnography }\end{array}$ \\
\hline $\begin{array}{l}\text { Student successfully participates in small } \\
\text { groups to complete projects. }\end{array}$ & $\begin{array}{l}\text { Goldberg et al., } 2003 \\
\text { Halpern, Yovanoff, Doren, \& Benz, } 1995\end{array}$ & $\begin{array}{l}\text { Qualitative Ethnography } \\
\text { Logistic Regression }\end{array}$ \\
\hline $\begin{array}{l}\text { Student participates in community } \\
\text { organizations, such as sports clubs or } \\
\text { organized social groups. }\end{array}$ & $\begin{array}{l}\text { Doren \& Benz, } 1998 \\
\text { Goldberg et al., } 2003\end{array}$ & $\begin{array}{l}\text { Logistic Regression } \\
\text { Qualitative Ethnography }\end{array}$ \\
\hline $\begin{array}{l}\text { Student participates in school teams, clubs, } \\
\text { or other groups. }\end{array}$ & Halpern et al., 1995 & Logistic Regression \\
\hline
\end{tabular}


Table I. (continued)

\begin{tabular}{cl}
\hline Specific behavior & \multicolumn{1}{c}{ Associated studies } \\
\hline Student successfully interacts with teachers, & Doren \& Benz, I998 \\
family, and other adults. & Goldberg et al., 2003 \\
& Halpern et al., I995 \\
& Liebert, Lutsky, \& Gottlieb, 1990
\end{tabular}

Construct 6: Goal setting and attainment Student defines the word "goal."

Student learns how to set a goal and make it happen.

Student talks about the importance of having goals.

Student expresses importance of having post-school goals that match his or her interests and skills.

Student identifies possible consequences of not setting goals.

Student sets goals that match his or her strengths and interests while taking into consideration what the family or community wants him or her to do.

Student creates short-term goals to attain long-term goals.

Student develops plans to attain his or her goals.

Student uses plans he or she develops to attain goals.

Student determines effectiveness of his or her plans to attain goals.

Student adjusts plans to attain goals if they do not work.

Student completes the first step of a goal, and then moves on to the next step.

Student attains one goal, and then moves on to the next goal.

Student attains at least one transition goal.

Construct 7: Employment

Student expresses wanting a job.

Student expresses wanting a job that matches his or her career interests and skills.

Student actively looks for a paid job.

Student demonstrates job-readiness skills such as being on time, completing work as assigned, or working cooperatively.
Raskind et al., 1999

Benz, Lindstrom, \& Yovanoff, 2000

Raskind et al., 1999

Sarver, 2000

Skinner, 2004

Raskind et al., 2002

Raskind et al., 1999

Gerber et al., 1992

Raskind et al., 2002

Raskind et al., 1999

Thoma \& Getzel, 2005

Raskind et al., 2002

Raskind et al., 1999

Fabian, Lent, \& Willis, 1998

Gerber et al., 1992

Raskind et al., 1999

Aune, 1991

Goldberg et al., 2003

Raskind et al., 2002

Raskind et al., 1999

Thoma \& Getzel, 2005

Benz et al., 2000

Goldberg et al., 2003

Goldberg et al., 2003

Raskind et al., 1999

Thoma \& Getzel, 2005

Raskind et al., 2002

Goldberg et al., 2003

Raskind et al., 2002

Raskind et al., 1999

Thoma \& Getzel, 2005

Gerber et al., 1992

Thoma \& Getzel, 2005

Benz et al., 2000

Benz et al., 2000

Fabian, 2007

Fabian et al., 1998

Fourqurean, Meisgeier, Swank, \& Williams, |991

McDonnall \& Crudden, 2009

Fabian, 2007

Leonard, D’Allura, \& Horowitz, 1999
Fabian et al., 1998

Heal \& Rusch, 1995
Study design

Logistic Regression

Qualitative Ethnography

Logistic Regression

Discriminate and Regression

Analysis

Correlation and Descriptive

Logistic Regression

Correlation and Descriptive

Correlation

Qualitative Constant Comparison

Qualitative

Correlation and Descriptive

Qualitative Case Study

Qualitative

Correlation and Descriptive

Qualitative

Qualitative

Correlation and Descriptive

Correlation Prediction

Qualitative Ethnography

Correlation and Descriptive

Descriptive

Qualitative Ethnography

Qualitative

Correlation and Descriptive

Qualitative

Logistic Regression

Qualitative Ethnography

Qualitative Ethnography

Correlation and Descriptive

Qualitative

Qualitative

Qualitative Ethnography

Qualitative

Correlation and Descriptive

Qualitative

Qualitative Ethnography

Qualitative

Logistic Regression

Logistic Regression

Discriminate Analysis

Correlation

Discriminate Analysis

Regression

Discriminate Analysis

Correlation

Hierarchical Regression

Logistic Regression 
Table I. (continued)

\begin{tabular}{|c|c|c|}
\hline Specific behavior & Associated studies & Study design \\
\hline $\begin{array}{l}\text { Student exhibits self-care habits, such as } \\
\text { personal hygiene or clothing choices, to } \\
\text { match career interests. }\end{array}$ & Heal \& Rusch, 1995 & Hierarchical Regression \\
\hline $\begin{array}{l}\text { Student successfully participates in a career } \\
\text { technology or job-training program. }\end{array}$ & $\begin{array}{l}\text { Baer et al., } 2003 \\
\text { Benz et al., } 2000 \\
\text { Fabian, } 2007 \\
\text { Fabian et al., } 1998 \\
\text { Flexer et al., } 201 \text { I } \\
\text { Hasazi, Johnson, Hasazi, Gordon, } \\
\text { \& Hull, I } 989 \\
\text { Rabren, Dunn, \& Chambers et al., } 2002 \\
\text { Shandra \& Hogan, } 2008\end{array}$ & $\begin{array}{l}\text { Logistic Regression } \\
\text { Logistic Regression } \\
\text { Discriminant analysis } \\
\text { Correlation } \\
\text { Logistic Regression } \\
\text { Correlation } \\
\text { Hierarchical Regression } \\
\text { Correlation }\end{array}$ \\
\hline $\begin{array}{l}\text { Student has an unpaid job, such as working } \\
\text { for a family member. }\end{array}$ & $\begin{array}{l}\text { Fabian et al., } 1998 \\
\text { Hasazi et al., } 1989\end{array}$ & $\begin{array}{l}\text { Correlation } \\
\text { Correlation }\end{array}$ \\
\hline $\begin{array}{l}\text { Student has an unpaid internship or } \\
\text { apprenticeship. }\end{array}$ & $\begin{array}{l}\text { Fabian et al., } 2007 \\
\text { Fabian et al., } 1998 \\
\text { Hasazi et al., } 1989 \\
\text { Shandra \& Hogan, } 2008\end{array}$ & $\begin{array}{l}\text { Discriminant analysis } \\
\text { Correlation } \\
\text { Correlation } \\
\text { Correlation }\end{array}$ \\
\hline Student has a paid job. & $\begin{array}{l}\text { Benz et al., I997 } \\
\text { Benz et al., } 2000 \\
\text { Doren \& Benz, I998 } \\
\text { Dunn \& Shumaker, I997 } \\
\text { Fabian, } 2007 \\
\text { Fourqurean et al., I99। } \\
\text { Hasazi et al., I985 } \\
\text { Hasazi et al., I989 } \\
\text { Lindstrom et al., 20I I } \\
\text { McDonnall, 20 I0 } \\
\text { Rabren et al., 2002 } \\
\text { Sitlington, Frank, \& Carson, } 1993\end{array}$ & $\begin{array}{l}\text { Logistic Regression } \\
\text { Logistic Regression } \\
\text { Logistic Regression } \\
\text { Chi-Square } \\
\text { Correlation } \\
\text { Multiple Regression } \\
\text { Correlation } \\
\text { Correlation } \\
\text { Qualitative Case Study } \\
\text { Multi-Level Modeling } \\
\text { Hierarchical Regression } \\
\text { Correlation and Descriptive }\end{array}$ \\
\hline \multicolumn{3}{|l|}{ Construct 8: Self-advocacy } \\
\hline $\begin{array}{l}\text { Student uses the Internet or other sources } \\
\text { to understand his or her disability, legal } \\
\text { rights, supports, or accommodations. }\end{array}$ & $\begin{array}{l}\text { Skinner, } 2004 \\
\text { Thoma \& Getzel, } 2005\end{array}$ & $\begin{array}{l}\text { Qualitative } \\
\text { Qualitative }\end{array}$ \\
\hline $\begin{array}{l}\text { Student identifies accommodations effective } \\
\text { for him or her. }\end{array}$ & Aune, 1991 & Descriptive \\
\hline $\begin{array}{l}\text { Student identifies accommodations ineffective } \\
\text { for him or her. }\end{array}$ & Thoma \& Getzel, 2005 & Qualitative \\
\hline $\begin{array}{l}\text { Student requests a new accommodation } \\
\text { when the first one was not effective. }\end{array}$ & Thoma \& Getzel, 2005 & Qualitative \\
\hline Student asks for support only when needed. & $\begin{array}{l}\text { Aune, 1991 } \\
\text { Skinner, } 2004\end{array}$ & $\begin{array}{l}\text { Descriptive } \\
\text { Qualitative }\end{array}$ \\
\hline Student talks during his or her IEP meeting. & $\begin{array}{l}\text { Aune, I99I } \\
\text { Halpern et al., } 1995 \\
\text { Portley, Martin, \& Hennessey, } 2012\end{array}$ & $\begin{array}{l}\text { Descriptive } \\
\text { Logistic Regression } \\
\text { Correlation Cluster Analysis }\end{array}$ \\
\hline $\begin{array}{l}\text { Student tells the IEP team his or her post- } \\
\text { school goals. }\end{array}$ & $\begin{array}{l}\text { Aune, } 1991 \\
\text { Halpern et al., } 1995 \\
\text { Portley et al., } 2012\end{array}$ & $\begin{array}{l}\text { Descriptive } \\
\text { Logistic Regression } \\
\text { Correlation Cluster Analysis }\end{array}$ \\
\hline $\begin{array}{l}\text { Student discusses his or her present level of } \\
\text { performance at the IEP meeting. }\end{array}$ & $\begin{array}{l}\text { Aune, } 1991 \\
\text { Halpern et al., } 1995 \\
\text { Portley et al., } 2012\end{array}$ & $\begin{array}{l}\text { Descriptive } \\
\text { Logistic Regression } \\
\text { Correlation Cluster Analysis }\end{array}$ \\
\hline $\begin{array}{l}\text { Student explains at the IEP meeting how } \\
\text { his or her course of study will assist in } \\
\text { achieving post-school goals. }\end{array}$ & $\begin{array}{l}\text { Aune, } 1991 \\
\text { Portley et al., } 2012\end{array}$ & $\begin{array}{l}\text { Descriptive } \\
\text { Correlation Cluster Analysis }\end{array}$ \\
\hline
\end{tabular}


Table I. (continued)

\begin{tabular}{|c|c|c|}
\hline Specific behavior & Associated studies & Study design \\
\hline Student leads his or her IEP meeting. & $\begin{array}{l}\text { Aune, } 1991 \\
\text { Halpern et al., } 1995 \\
\text { Portley et al., } 2012\end{array}$ & $\begin{array}{l}\text { Descriptive } \\
\text { Logistic Regression } \\
\text { Correlation Cluster Analysis }\end{array}$ \\
\hline \multicolumn{3}{|l|}{ Construct 9: Supports } \\
\hline $\begin{array}{l}\text { Student distinguishes between individuals } \\
\text { who are a positive source of support from } \\
\text { those who are not. }\end{array}$ & $\begin{array}{l}\text { Goldberg et al., } 2003 \\
\text { Raskind, I } 999 \\
\text { Raskind et al., } 2002\end{array}$ & $\begin{array}{l}\text { Qualitative Ethnography } \\
\text { Hierarchical Regression } \\
\text { Qualitative }\end{array}$ \\
\hline $\begin{array}{l}\text { Student identifies situations when support } \\
\text { people are needed. }\end{array}$ & $\begin{array}{l}\text { Benz et al., } 2000 \\
\text { Goldberg et al., } 2003 \\
\text { Greenbaum et al., } 1995 \\
\text { Liebert et al., I } 990 \\
\text { Madaus, } 2006 \\
\text { Raskind et al., } 2002 \\
\text { Sarver, } 2000 \\
\text { Thoma \& Getzel, } 2005\end{array}$ & $\begin{array}{l}\text { Logistic Regression } \\
\text { Qualitative Ethnography } \\
\text { Qualitative Descriptive } \\
\text { Discriminate Regression } \\
\text { Qualitative Inductive } \\
\text { Qualitative } \\
\text { Correlation } \\
\text { Qualitative }\end{array}$ \\
\hline $\begin{array}{l}\text { Student identifies the support person } \\
\text { needed for a specific situation. }\end{array}$ & $\begin{array}{l}\text { Benz et al., } 2000 \\
\text { Gerber et al., } 1992 \\
\text { Goldberg et al., } 2003 \\
\text { Greenbaum et al., } 1995 \\
\text { Raskind, I } 999 \\
\text { Raskind et al., } 2002 \\
\text { Skinner, } 2004\end{array}$ & $\begin{array}{l}\text { Logistic Regression } \\
\text { Qualitative Ethnography } \\
\text { Qualitative Ethnography } \\
\text { Qualitative Descriptive } \\
\text { Correlation and Descriptive } \\
\text { Qualitative } \\
\text { Qualitative }\end{array}$ \\
\hline $\begin{array}{l}\text { Student accepts help from support people } \\
\text { when offered. }\end{array}$ & $\begin{array}{l}\text { Doren \& Benz, I } 998 \\
\text { Gerber et al., } 1992 \\
\text { Greenbaum et al., } 1995 \\
\text { Lindstrom et al., 201 I } \\
\text { Raskind et al., } 2002\end{array}$ & $\begin{array}{l}\text { Logistic Regression } \\
\text { Qualitative Case Study } \\
\text { Qualitative Descriptive } \\
\text { Qualitative Case Study } \\
\text { Qualitative }\end{array}$ \\
\hline $\begin{array}{l}\text { Student only uses support people when } \\
\text { needed, not to get out of doing things. }\end{array}$ & $\begin{array}{l}\text { Gerber et al., } 1992 \\
\text { Goldberg et al., } 2003\end{array}$ & $\begin{array}{l}\text { Qualitative Ethnography } \\
\text { Qualitative Ethnography }\end{array}$ \\
\hline $\begin{array}{l}\text { Student maintains the support network by } \\
\text { showing appreciation or reciprocity. }\end{array}$ & $\begin{array}{l}\text { Gerber et al., } 1992 \\
\text { Raskind et al., } 2002\end{array}$ & $\begin{array}{l}\text { Qualitative Ethnography } \\
\text { Qualitative }\end{array}$ \\
\hline \multicolumn{3}{|l|}{ Construct 10: Utilization of resources } \\
\hline $\begin{array}{l}\text { Student actively seeks people to help with a } \\
\text { situation when the current support people } \\
\text { cannot help. }\end{array}$ & $\begin{array}{l}\text { Gerber et al., } 1992 \\
\text { Raskind et al., } 2002\end{array}$ & $\begin{array}{l}\text { Qualitative Ethnography } \\
\text { Qualitative }\end{array}$ \\
\hline $\begin{array}{l}\text { Student uses available support people at } \\
\text { school or work. }\end{array}$ & $\begin{array}{l}\text { Gerber et al., } 1992 \\
\text { Lindstrom et al., } 201 \text { I } \\
\text { Skinner, } 2004\end{array}$ & $\begin{array}{l}\text { Qualitative Ethnography } \\
\text { Qualitative Case Study } \\
\text { Qualitative }\end{array}$ \\
\hline $\begin{array}{l}\text { Student uses the Internet to access } \\
\text { information for possible support services } \\
\text { or community agencies. }\end{array}$ & $\begin{array}{l}\text { Gerber et al., I } 992 \\
\text { Goldberg et al., } 2003 \\
\text { Liebert et al., } 1990 \\
\text { Thoma \& Getzel, } 2005\end{array}$ & $\begin{array}{l}\text { Qualitative Ethnography } \\
\text { Qualitative Ethnography } \\
\text { Discriminate Regression } \\
\text { Qualitative }\end{array}$ \\
\hline $\begin{array}{l}\text { Student seeks assistance from community } \\
\text { agencies. }\end{array}$ & $\begin{array}{l}\text { Gerber et al., I } 992 \\
\text { Goldberg et al., } 2003 \\
\text { Liebert et al., } 1990 \\
\text { Thoma \& Getzel, } 2005\end{array}$ & $\begin{array}{l}\text { Qualitative Ethnography } \\
\text { Qualitative Ethnography } \\
\text { Discriminate Regression } \\
\text { Qualitative }\end{array}$ \\
\hline
\end{tabular}

Note. IEP = Individualized Education Program.

disability and learning to confront and avoid specific situations based on this knowledge (Gerber et al., 1992). Students need to explain their disability in a variety of ways to ensure others with and without disability-related knowledge will understand the accommodations that students will need to successfully complete assigned tasks (Aune, 1991; Gerber et al., 1992; Gerber, Price, Mulligan, \& Shessel, 2004;
Higgins et al., 2002; Raskind et al., 1999). Students need to practice explaining their disability and asking for accommodations (Aune, 1991; Gerber et al., 1992; Greenbaum et al., 1995; Higgins et al., 2002; Thoma \& Getzel, 2005). Students may obtain disability information from a variety of settings, including the Internet, doctors, teachers, and family members (Thoma \& Getzel, 2005). 


\section{Persistence}

Individuals with disabilities who engage in employment and postsecondary education express the importance of persistence in all aspects of life (Goldberg et al., 2003; Greenbaum et al., 1995; Raskind et al., 1999; Sarver, 2000; Skinner, 2004), and they keep attempting to attain their goals in spite of barriers (Raskind et al., 2002). Individuals with disabilities who experienced success in postsecondary educational settings began college knowing they would spend more hours studying and completing required assignments than other students and began this behavior in high school (Skinner, 2004). They did not give up when faced with adversity, but shifted goals when necessary to accomplish them (Gerber et al., 1992; Goldberg et al., 2003; Greenbaum et al., 1995; Sarver, 2000; Skinner, 2004). Successful college students with disabilities learned to use a variety of flexible strategies to continue their college pursuit, including changing professors, classes, majors, colleges, and seeking individuals for assistance (Goldberg et al., 2003; Sarver, 2000), and their own tenacity enabled them to complete the tasks needed to graduate (Greenbaum et al., 1995).

\section{Proactive Involvement}

Individuals who are successful in the areas of education and employment after high school actively interact with others positively in a variety of settings (Goldberg et al., 2003; Halpern, Yovanoff, Doren, \& Benz, 1995; Liebert, Lutsky, \& Gottlieb, 1990). Doren and Benz (1998) found males and females who used the self-family-friend network to obtain employment were, respectively, 2.33 and 3.77 times more likely to be employed after high school. Goldberg et al. (2003) found proactivity predicted post-school involvement in employment and education by stating that these individuals with disabilities "were engaged in the world around them, politically, economically, and socially. They participated in community activities and took an active role in their families, neighborhoods, and friendship groups" (p. 226).

\section{Goal Setting and Attainment}

Adults with disabilities who participate in employment and postsecondary education set explicit long- and shortterm goals that define what can be accomplished, and then focus on attaining them (Gerber et al., 1992). Students who set goals and commit to attaining them are more successful academically (Sarver, 2000). Raskind et al. (1999) reported goal setting along with other attributes predicted post-school employment and education better than IQ, academic achievement, social economic status, and ethnicity. Individuals with disabilities who participated in postschool employment and education learned to break down large goals into smaller manageable pieces and accomplish the small goals in a sequential order (Thoma \& Getzel, 2005). Individuals with disabilities with positive postschool outcomes in employment and education set realistic goals for themselves that are meaningful (Benz et al., 2000), make a plan to achieve the goals (Gerber et al., 1992; Thoma \& Getzel, 2005), and then act in purposeful ways to attain the goals (Skinner, 2004). Gerber et al. (1992) and Goldberg et al. (2003) stressed the implementation of the plan to achieve goals as important, while remaining flexible with the plan without straying completely away from the goals. Portley, Martin, and Hennessey (2012) found students in districts that involve students in setting their postsecondary goals have greater post-school employment and education outcomes. Benz et al. (2000) indicated students who completed four or more of their transition goals were more likely to be engaged after high school than students who completed fewer goals or none at all. Successful college students set high goals for themselves, while considering the desires of their families (Thoma \& Getzel, 2005). Goldberg et al. (2003) found the goals of individuals who did not engage in employment or post-school education lacked a plan of achievement and were not specific or flexible. It is imperative that individuals set goals that are "concrete, realistic, and attainable" (Goldberg et al., 2003, p. 228). Active involvement in IEP meetings is an excellent means for students with disabilities to set appropriate goals and determine the smaller steps needed to attain the goal (Aune, 1991).

\section{Employment}

To experience successful post-school employment, students must first express a desire to obtain a job, and then actively seek a position using effective job search skills (Benz et al., 2000; Benz, Yovanoff, \& Doren, 1997; Fabian, 2007). Participation by students with disabilities in work-study and vocational education (Baer et al., 2003; Flexer, Daviso, Baer, Queen, \& Meindl, 2011), job internships (Fabian, Lent, \& Willis, 1998), or mentoring (Shandra \& Hogan, 2008) increased the likelihood of full-time employment. Students with disabilities who obtained employment during the high school years were more likely to be employed after high school (Doren \& Benz, 1998; Dunn \& Shumaker, 1997; Fabian, 2007; Fourqurean, Meisgeier, Swank, \& Williams, 1991; Hasazi, Gordon, \& Roe, 1985; Hasazi, Johnson, Hasazi, Gordon, \& Hull, 1989; Lindstrom et al., 2011; McDonnall, 2010; Portley et al., 2012; Rabren, Dunn, \& Chambers, 2002; Sitlington, Frank, \& Carson, 1993). Students who had previous paid employment experiences are more able to find future jobs that match their skills and interests, and are more able to seek and find jobs in the community (Fourqurean et al., 1991; McDonnall \& Crudden, 2009). Students demonstrate job-readiness skills by attending school on time, getting along with others, and managing personal hygiene (Heal \& Rusch, 1995; Leonard, D’Allura, \& Horowitz, 1999). 


\section{Self-Advocacy}

Students must understand their disability, their strengths, and weaknesses and how these affect them before they can learn to be advocates for themselves (Aune, 1991). Selfadvocates understand their disability, know their legal rights, and can tell others, including those in leadership positions, about their rights, needs, and goals in an assertive manner to obtain support, accommodations, and assistance (Skinner, 2004; Test, Fowler, Wood, Brewer, \& Eddy, 2005). Unfortunately, many students with disabilities do not have the opportunity while in high school to learn to advocate for themselves (Aune, 1991). To learn the necessary skills, students need to first understand their disability, strengths, and weaknesses, practice self-advocacy skills in high school to request accommodations and supports, and to be actively involved in IEP meetings (Aune, 1991). After reviewing the self-advocacy literature, Test et al. (2005) concluded that self-advocacy involves three essential components with the foundational skills being self-knowledge and knowledge of rights and the third element being the ability to communicate their knowledge and rights effectively.

Self-advocacy is a key element for student participation in postsecondary education, and students must be able to explain their disability rights and responsibilities to those who may not have a working knowledge of disabilityrelated issues (Skinner, 2004). Students with disabilities with more positive post-school outcomes in the areas of employment and education use multiple sources to gather information about their disability, including the Internet, educators, and support people (Skinner, 2004; Thoma \& Getzel, 2005). They must be able to express which accommodations best enable them to achieve in various situations (Aune, 1991). The IEP meeting provides an opportunity for students with disabilities to practice self-advocacy skills when students take an active role in conducting and leading the meeting (Aune, 1991). Halpern et al. (1995) and Portley et al. (2012) found students who actively participate in transition planning IEP meetings had better employment and/or postsecondary education outcomes than students who attended school in districts that had low levels of student involvement in IEP transition planning meetings.

\section{Supports}

Use of support systems has, in part, contributed to improved outcomes of adults with disabilities 20 years after they graduated from high school (Raskind et al., 1999). Greenbaum et al. (1995) and Skinner (2004) found achievement of college students with disabilities was related to the support, advice, and encouragement that came from significant others, including family members, close friends, faculty, or academic support providers. Benz et al. (2000) found adolescents with disabilities needed educators who encouraged students' efforts, provided gentle pushing, and acknowledged their accomplishments. Lindstrom et al. (2011) found young women with disabilities who successfully participated in employment had family members who strongly supported their goals and communicated clear expectations for them. Students with disabilities who have a group of positive individuals for support, including mentors, other students with disabilities, friends, and coworkers are more likely to participate in employment and education after high school (Gerber et al., 1992; Goldberg et al., 2003; Liebert et al., 1990; Madaus, 2006; Raskind et al., 2002; Sarver, 2000; Skinner, 2004; Thoma \& Getzel, 2005). Students need to recognize situations when they need support, identify a support person who can offer the assistance in the specific situation (Gerber et al., 1992; Goldberg et al., 2003), accept and use the support when available or offered, and only use the support when needed (Gerber et al., 1992; Raskind et al., 1999). Students need to maintain their support groups by showing appreciation and reciprocity (Goldberg et al., 2003).

\section{Utilization of Resources}

Individuals with disabilities who participate in post-school employment and education may actively seek people and resources outside their immediate network to help with a present need (Gerber et al., 1992; Raskind et al., 2002). High school students need to learn how to seek assistance and practice seeking support at school from coaches, secretaries, school counselors, teachers, or transition specialists (Gerber et al., 1992; Lindstrom et al., 2011; Skinner, 2004). Individuals who are engaged in post-school employment and education also use the Internet to obtain information and seek assistance from possible support services or community agencies (Gerber et al., 1992; Goldberg et al., 2003; Liebert et al., 1990; Thoma \& Getzel, 2005).

\section{Discussion}

We undertook this review of the transition education research literature to build a comprehensive set of behavioral constructs associated with engagement in postsecondary employment and education. An iterative review process yielded 10 constructs associated with post-school education and employment of former high school students with mild to moderate disabilities. Students must have knowledge of their own personal strengths and limitations in multiple settings, act upon this knowledge, and consider their strengths and limitations when making decisions. Students need to understand and be able to explain their disability to others. Having a paid job during the high school years is positively associated with employment after high school. Students with disabilities need to learn how to set and attain goals. This requires breaking down large goals to make smaller 
goals, and making a plan to implement the smaller goals. Persistence is an important behavior for students with disabilities to exhibit while using a variety of strategies to complete tasks and attain goals. Students with disabilities need to use a support network to overcome barriers and assist with attaining goals. Students with disabilities need to understand their rights and responsibilities and be able to advocate for themselves. When those in the student's immediate support group cannot help with a specific task, students need to utilize resources for assistance.

This review extends the current literature by identifying nonacademic teachable behaviors and skills known to indicate post-school employment and education based on research from multiple sources and study designs. Test, Fowler, et al. (2009) identified practices to improve student transition skills and Test, Mazzotti, et al. (2009) specifically included only correlational research to identify 16 in-school predictors of post-school education, employment, and/or independent living with only four of the predictor categories, including teachable student behaviors. Just as academic achievement assessments determine present levels of reading, spelling, and math calculation, there is a need for an assessment to identify nonacademic skills students need to learn while in high school to increase their likelihood for post-high school employment and education.

\section{Limitations and Suggestions for Future Research}

As with all studies, there are some limitations. First, the results cannot be generalized to all students due to our focus on former high school students with mild to moderate disabilities. To increase the generalizability of the results, the methods used in this study would need to be replicated with research reporting indicators of post-school employment and education for individuals without disabilities and those with significant intellectual disabilities. The identified behaviors could then be compared and combined to determine commonalities and differences.

Second, we considered the peer-review process used by journals as sufficiently rigorous to determine whether a study had adequate quality to be included. This could, however, be considered a limitation because a quality indicator checklist for specific research designs was not utilized. Use of this type of checklist was not of concern to us because future assessment development studies will determine the extent that identified behaviors and clusters of the behaviors predict student employment and enrollment in further education, and our purpose was to assemble the largest pool of behaviors possible.

Third, each of our constructs is supported by multiple research approaches; however, some specific behaviors are only supported by qualitative research methods, which may not be generalizable to all individuals with disabilities due to the small sample sizes used in some of the studies. This possible concern is alleviated because quantitative studies with larger sample sizes identified other behaviors within the construct. Qualitative studies identified behaviors from a rich environmental context and expanded the breadth of behaviors and helped produce an inclusive study not limited by type of study design or analysis.

Finally, this review and analysis of transition research identified and defined 10 constructs and associated behaviors that contributed to the post-school employment and education of high school students with mild to moderate disabilities. The next steps will be to develop items, create a scoring system, and write multiple versions of an assessment based on these constructs; have students, family members, and educators complete the assessment; conduct an exploratory factor analysis to identify the factor structure and to eliminate duplicative or unnecessary items, to determine its reliability; and then to undertake a follow-along study to determine the relationship between specific items and actual post-school outcomes. Using such a transition assessment will yield a profile of student strengths and identify what students still need to learn while in high school. Using the results of the described assessment, educators will need to develop opportunities to teach students the skills needed for post-school employment and education.

\section{Student Behaviors for Post-School Employment and Education Assessment}

Special education law requires transition assessments be used to facilitate identification of transition goals. Unfortunately, no existing transition assessment uses test items verified by research as associated with post-high school education and employment of former students with mild and moderate disabilities (McConnell, 2012). Special educators need to write annual transition goals using behaviors that when learned will increase students' likelihood of postsecondary employment and education, but no transition assessment has been validated for this purpose (McConnell, 2012). Transition assessments need to identify the behaviors students have already mastered and those that students still need to learn. By undertaking this literature review, we have developed a comprehensive set of constructs, defined them, and provided examples of student behaviors necessary to create a new transition assessment that educators, students, and family members can use to identify behaviors that students need to acquire to increase their likelihood of post-school transition into education or employment.

\section{Implications for Practice}

The construct-building process described in this article will enable us to create a transition assessment to identify meaningful annual transition goals that when learned will 
increase students' likelihood of participation in postsecondary employment and education. Development of this assessment begins with defining clusters of student behaviors found in the transition research literature associated with successful involvement in postsecondary employment and education for students with disabilities. The completed assessment will provide graphic profile results and prioritized suggestions for annual transition goals. The items for this assessment, called the Transition Assessment and Goal Generator (TAGG), will be organized into clusters of nonacademic behaviors identified from the research literature as being associated with post-school employment or education participation. To develop this assessment, we will follow the procedures established in the Standards for Educational and Psychological Testing (American Educational Research Association, American Psychological Association, \& National Council on Measurement in Education, 1999), which involves (a) building the constructs that will be measured, (b) developing the items and scoring methods, (c) field-testing and examining the factor structure of the assessment, (d) and then developing the profile and use of the results. This study built the constructs to be used in developing the TAGG.

\section{Declaration of Conflicting Interests}

The author(s) declared no potential conflicts of interest with respect to the research, authorship, and/or publication of this article.

\section{Funding}

The author(s) disclosed receipt of the following financial support for the research, authorship, and/or publication of this article: This research was supported by a grant from the Institute of Education Sciences, National Center for Special Education Research, Grant Award Number R324A100246.

\section{References}

American Educational Research Association, American Psychological Association, \& National Council on Measurement in Education. (1999). Standards for educational and psychological testing. Washington, DC: American Psychological Association.

Aune, E. (1991). A transition model for postsecondary-bound students with learning disabilities. Learning Disabilities Research \& Practice, 6, 177-187.

Baer, R. M., Flexer, R. W., Beck, S., Amstutz, N., Hoffman, L., Brothers, J., \& Zechman, C. (2003). A collaborative followup study on transition service utilization and post-school outcomes. Career Development for Exceptional Individuals, 26, $7-25$

Benz, M., Lindstrom, L., \& Yovanoff, P. (2000). Improving graduation and employment outcomes of students with disabilities: Predictive factors and student perspectives. Exceptional Children, 66, 509-529.
Benz, M., Yovanoff, P., \& Doren, B. (1997). School-to-work components that predict postschool success for students with and without disabilities. Exceptional Children, 63, 151-165.

Doren, B., \& Benz, M. (1998). Employment inequality revisited: Predictors of better employment outcomes for young women with disabilities in transition. Journal of Special Education, $31,425-442$.

Dunn, C., \& Shumaker, L. (1997). A follow-up study of former special education students from a rural and urban county school system. Career Development for Exceptional Individuals, 20, 43-54.

Fabian, E. (2007). Urban youth with disabilities: Factors affecting transition employment. Rehabilitation Counseling Bulletin, 50, 130-138.

Fabian, E., Lent, R., \& Willis, S. (1998). Predicting work transition outcomes for students with disabilities: Implications for counselors. Journal of Counseling \& Development, 76, 311-316.

Flexer, R. W., Daviso, A. W., Baer, R. M., Queen, R. M., \& Meindl, R. S. (2011). An epidemiological model of transition and post-school outcomes. Career Development for Exceptional Individuals, 34, 83-94.

Fourqurean, J., Meisgeier, C., Swank, P., \& Williams, R. (1991). Correlates of postsecondary employment outcomes for young adults with learning disabilities. Journal of Learning Disabilities, 24, 400-405.

Gerber, P., Ginsberg, R., \& Reiff, H. (1992). Identifying alterable patterns in employment success for highly successful adults with learning disabilities. Journal of Learning Disabilities, 25, 475-487.

Gerber, P., Price, L., Mulligan, R., \& Shessel, I. (2004). Beyond transition: A comparison of the employment experiences of American and Canadian adults with LD. Journal of Learning Disabilities, 37, 283-291.

Goldberg, R., Higgins, E., Raskind, M., \& Herman, K. (2003). Predictors of success in individuals with learning disabilities: A qualitative analysis of a 20-year longitudinal study. Learning Disabilities Research \& Practice, 18, 222-236.

Greenbaum, B., Graham, S., \& Scales, W. (1995). Adults with learning disabilities: Education and social experiences during college. Exceptional Children, 61, 460-471.

Halpern, A., Yovanoff, P., Doren, B., \& Benz, M. (1995). Predicting participation in postsecondary education for school leavers with disabilities. Exceptional Children, 62, 151-164.

Hasazi, S., Gordon, L., \& Roe, C. (1985). Factors associated with the employment status of handicapped youth exiting high school from 1979 to 1983. Exceptional Children, 51, 455-469.

Hasazi, S., Johnson, R., Hasazi, J., Gordon, L., \& Hull, M. (1989). Employment of youth with and without handicaps following high school: Outcomes and correlates. Journal of Special Education, 23, 243-255.

Heal, L. W., \& Rusch, F. R. (1994). Prediction of residential independence of special education high school students. Research in Developmental Disabilities, 15, 223-243. 
Heal, L. W., \& Rusch, F. R. (1995). Predicting employment for students who leave special education high school programs. Exceptional Children, 61, 472-487.

Higgins, E., Raskind, M., Goldberg, R., \& Herman, K. (2002). Stages of acceptance of a learning disability: The impact of labeling. Learning Disability Quarterly, 25, 3-18.

Individuals With Disabilities Education Improvement Act of 2004, P.L. 108-446, 20 U.S.C. $§ 1400$ et seq.

Institute of Education Sciences. (2012). Request for applications (Special education research grants CFDA Number: 84.324A). Retrieved from http://ies.ed.gov/funding/ncser rfas/ncser_transition.asp

Juan, C. (2008). The transition success assessment: Determining social validity and reliability (Unpublished doctoral dissertation). University of Oklahoma, Norman.

Kochhar-Bryant, C. A., Shaw, S., \& Izzo, M. (2007). What every teacher should know about transition and IDEA 2004. Boston, MA: Pearson.

Leonard, R., D’Allura, T., \& Horowitz, A. (1999). Factors associated with employment among persons who have a vision impairment: A follow-up of vocational placement referrals. Journal of Vocational Rehabilitation, 12, 33-43.

Liebert, D., Lutsky, L., \& Gottlieb, A. (1990). Postsecondary experiences of young adults with severe physical disabilities. Exceptional Children, 57, 56-63.

Lindstrom, L., Doren, B., \& Miesch, J. (2011). Waging a living: Career development and long-term employment outcomes for young adults with disabilities. Exceptional Children, 77, 423-434.

Madaus, J. (2006). Improving the transition to career for college students with learning disabilities: Suggestions from graduates. Journal of Postsecondary Education and Disability, 19, 85-93.

McConnell, A. (2012). The relationship among academic GPA, the $T A G G$, and students with mild to moderate disabilities (Unpublished doctoral dissertation). University of Oklahoma, Norman.

McDonnall, M. C. (2010). Factors predicting post-high school employment for young adults with visual impairments. Rehabilitation Counseling Bulletin, 54, 36-45.

McDonnall, M. C., \& Crudden, A. (2009). Factors affecting the success of transition-aged youths with visual impairments. Journal of Visual Impairments \& Blindness, 103, 329-341.

McNulty, M. (2003). Dyslexia and the life course. Journal of Learning Disabilities, 36, 363-381.

Portley, J. C., Martin, J. E., \& Hennessey, M. N. (2012). Impact of student involvement in IEP meetings and other transition education factors on transition outcome indicators 13 and 14. Manuscript submitted for publication.

Rabren, K., Dunn, C., \& Chambers, D. (2002). Predictors of posthigh school employment among young adults with disabilities. Career Development for Exceptional Individuals, 25, 25-40.

Raskind, M., Goldberg, R., Higgins, E., \& Herman, K. (1999). Patterns of change and predictors of success in individuals with learning disabilities: Results from a twenty-year longitudinal study. Learning Disabilities Research \& Practice, 14, 35-49.

Raskind, M., Goldberg, R., Higgins, E., \& Herman, K. (2002). Teaching "life success" to students with LD: Lessons learned from a 20-year study. Intervention in School and Clinic, 37, 201-208.

Rusch, F. R., Hughes, C., Agran, M., Martin, J. E., \& Johnson, J. R. (2009). Toward self-directed learning, post-high school placement, and coordinated support: Constructing new transition bridges to adult life. Career Development for Exceptional Individuals, 32, 53-59.

Sands, D. J., Spencer, K. C., Gliner, J., \& Swaim, R. (1999). Structural equation modeling of student involvement in transitionrelated actions: The path of least resistance. Focus on Autism and Other Developmental Disabilities, 14, 35.

Sarver, M. (2000). A study of the relationship between personal and environmental factors bearing on self-determination and the academic success of university students with learning disabilities (Unpublished doctoral dissertation). University of Florida, Gainesville. (UMI Number: 9984478)

Shandra, C., \& Hogan, D. (2008). School-to-work program participation and the post-high school employment of young adults with disabilities. Journal of Vocational Rehabilitation, 29, 117-130.

Sitlington, P., Frank, A., \& Carson, R. (1993). Adult adjustment among high school graduates with mild disabilities. Exceptional Children, 59, 221-233.

Skinner, M. (2004). College students with learning disabilities speak out: What it takes to be successful in postsecondary education. Journal of Postsecondary Education and Disability, 17, 91-104.

Spradley, J. P. (1979). The ethnographic interview. New York, NY: Holt, Rinehart \& Winston.

Test, D. W., Aspel, N. P., \& Everson, J. M. (2006). Transition methods for youth with disabilities. Upper Saddle River, NJ: Pearson.

Test, D. W., Fowler, C. H., Richter, S. M., White, J., Mazzotti, V., Walker, A., \& Kortering, L. (2009). Evidence-based practices in secondary transition. Career Development for Exceptional Individuals, 32, 115-128.

Test, D. W., Fowler, C. H., Wood, W. M., Brewer, D. M., \& Eddy, S. (2005). A conceptual framework of self-advocacy for students with disabilities. Remedial and Special Education, 26, 43-54.

Test, D. W., Mazzotti, V. L., Mustian, A. L., Fowler, C. H., Kortering, L. J., \& Kohler, P. H. (2009). Evidence-based secondary transition predictors for improving post-school outcomes for students with disabilities. Career Development for Exceptional Individuals, 32, 160-181.

Thoma, C., \& Getzel, E. (2005). "Self-determination is what it's all about": What post-secondary students with disabilities tell us are important considerations for success. Education and Training in Developmental Disabilities, 40, 234-242. 